\title{
Atomistic modeling of growth and coalescence of helium nano-bubbles in
}

\section{tungsten}

\author{
R.D. Smirnov ${ }^{\mathrm{a}, *}$, S.I. Krasheninnikov ${ }^{\mathrm{a}, \mathrm{b}}$, J. Guterl $^{\mathrm{a}}$ \\ ${ }^{a}$ University of California San Diego, La Jolla, California 92093, USA \\ ${ }^{\mathrm{b}}$ Nuclear Research National University MEPhI, Moscow 115409, Russia
}

\begin{abstract}
The mechanisms of growth and coalescence of helium nano-bubbles in tungsten are investigated using molecular dynamics simulations. It is shown that crystal symmetries and governed by them properties of dislocations, generated by the growing nano-bubbles, are responsible for main nano-bubble features revealed, including non-spherical shape and anisotropy of surrounding stress field. The transport of helium atoms in non-uniform stress field is simulated at different temperatures and the transport coefficients are determined. The implications of the considered dislocation and helium dynamics on nucleation and growth of bubbles in tungsten with implanted helium are discussed.
\end{abstract}

PACS: 52.40.Hf, 61.72.Qq, 66.30.Pa, 83.10.Rs

PSI-21 Keywords: Tungsten, Helium, Bubble, Molecular dynamics

Corresponding Author Address: 9500 Gilman Dr., MAE, La Jolla, CA 92093-0411, USA

Corresponding Author e-mail: rsmirnov@ucsd.edu

Presenting Author: Prof. Sergei Krasheninnikov

Presenting Authore-mail:skrash@mae.ucsd.edu 


\section{Introduction}

At present, tungsten (W) is considered as a primary candidate material for high heat load plasma-facing components in next generation magnetic fusion devices, in particular ITER, due to its low plasma sputtering yield and favorable thermo-mechanical characteristics. However, it is anticipated that morphology and properties of plasma-exposed tungsten surfaces can be substantially altered by various plasma-surface interaction processes in fusion environment. It was experimentally observed that filamentary nano-structures, called fuzz, can grow on tungsten surfaces irradiated with plasma containing helium (He) [1,2]. Although, the mechanism of the fuzz growth is not yet understood, experiments show that formation of helium nano-bubbles in tungsten surface layer always precedes fuzz creation and that such bubbles are also formed inside the fuzz filaments. Some existing models of fuzz growth [3-5], thus, are based on the assumption that the creation and dynamics of helium bubbles in tungsten are responsible for fuzz formation. In this work, we aim clarification of some fundamental mechanisms involved in growth of helium bubbles in tungsten.

\section{General simulation procedures}

To simulate the processes involving transport of helium atoms and growth of helium bubbles in tungsten we employed the LAMMPS molecular dynamics (MD) code [6], which allows modeling of wide variety of atomic interactions and arrangements. In the simulations of tungsten-helium atomic systems we used for W-W interactions the multi-body Embedded Atom Model Finnis-Sinclair potential produced by Ackland and Mendelev [7]. The W-He atomic interactions were simulated using tabulated ab initio potential created by Juslin [8]. The potential was designed to well reproduce properties of helium interstitials and clusters in tungsten lattice. Finally, for the He-He interactions the long established Beck's empirical 
potential $[9,10]$ was used. The W-W and He-He potentials were cut-off at distance $0.41148 \mathrm{~nm}$ and the $\mathrm{W}-\mathrm{He}$ potential at $0.32500 \mathrm{~nm}$. The MD simulations of various geometries considered below were performed at different fixed values of temperature, treated as an externally controlled system parameter. As the number of atoms in the modeled systems was limited and varied from several thousand to a few hundred thousand, we used Berendsen velocity rescaling technique to reduce temperature fluctuations of the simulated ensemble. This was also necessary to accommodate dissipation of energy introduced into system by implanted helium atoms. The tungsten structures simulated in this work were initially constructed as bcc monocrystals with the lattice constant $a=0.31652 \mathrm{~nm}$ and then relaxed using system energy and inter-atomic force minimization methods. The simulation timestep was set equal $1 \mathrm{fs}$ and the calculations proceeded to model system evolution typically for $\sim 10-100 \mathrm{~ns}$.

\section{Growth of helium bubble in tungsten}

To investigate basic processes occurring in tungsten lattice during growth of helium bubbles we simulate the growth of a bubble in a monocrystal tungsten sphere of radius $25 a=7.913 \mathrm{~nm}$ by inserting helium atoms into a small spherical volume of radius $0.1 a$ in the center of the sphere with rate 1 atom/ps in bunches of 10 atoms. The energy of new helium atoms is minimized at each insertion to avoid atoms overlapping and then the whole system is evolved for $10 \mathrm{ps}$ till the insertion repeats. The external surface of the tungsten sphere was treated as free with no constrains on its modification. The spherical shape of the simulated tungsten nano-structure was chosen to eliminate any effects related to preferred directions other than the crystallographic ones. The simulation of the bubble growth inside the sphere continued until the bubble burst through the tungsten surface. 
An example of the helium bubble grown in tungsten at $1000 \mathrm{~K}$ is shown in Fig.1. The figure shows the reconstructed bubble surface, encompassing helium atoms, in orthographic projection on (111) crystallographic plane. The $\mathrm{X}, \mathrm{Y}$, and $\mathrm{Z}$ axes, which projections onto (111) plane are plotted in the figure, correspond to [100], [010], and [001] crystallographic directions in bcc lattice, respectively. At the stage displayed, the bubble contains 1000 helium atoms and has size $\sim 2.5 \mathrm{~nm}$. As one can see, the helium bubble is non-spherical with a shape resembling an irregular polyhedron with identifiable vertexes, edges and faces. The straight lines showing the axes projections are also representing intersection lines of the (111) plane of the figure with $\{\overline{1} 10\}$ system of planes, which are perpendicular to (111). The planes of types $\{11 \overline{2}\}$ and $\{12 \overline{3}\}$, not shown in the figure, correspondingly form angles of $30^{\circ}$ and $\approx 19.11^{\circ}$ with $\{\overline{1} 10\}$ planes and are also perpendicular to the plane of Fig.1. The planes of types $\{\overline{1} 10\}$, $\{11 \overline{2}\}$, and $\{12 \overline{3}\}$ are the slip planes in bcc crystals with $\langle 111\rangle$ slip directions, which form systems of very similar critically resolved shear stress for slip [11].

We observe from the bubble growth simulations that the faces of the bubble polyhedron have tendency to be parallel to the slip planes with the lower indexes; and its edges align parallel to the slip directions $\langle 111\rangle$. To explain these tendencies we need to consider the mechanism of growth of the bubbles in tungsten. In Fig. 1 the gray circles show tungsten atoms in the vicinity of the bubble not residing in regular bcc positions according to Common Neighbor Analysis performed. Due to rather high temperature of the simulated system, $T=1000 \mathrm{~K}$, the shown atomic positions were averaged over $10 \mathrm{ps}$ time interval to reduce noise associated with thermal motion. One can see, that the shown tungsten atoms form a few dislocation loops connected to the surface of the helium bubble. The loops are anchored at the bubble polyhedron vertexes and are formed initially along its edges. In the elastic stress field created by the bubble and other dislocations, the dislocations arc on a slip plane outward from the bubble. The analysis performed using Dislocation Extraction 
Algorithm [12] shows that the Burger's vectors of the formed dislocations are aligned with $\langle 111\rangle$ directions and have magnitude $b=a \sqrt{3} / 2$, as should be expected in a bcc system. As the bubble grows, the length and number of the dislocations increases. Two or more dislocations can be anchored at the same vertex that creates conditions for dislocation reactions. In particular, if the dislocations surrounding one or more faces of the bubble polyhedron have the same Burger's vector, they can form a prismatic loop, which has a polygonal shape with the sides laying in different slip planes. In sufficiently strong elastic shear stress field, created by the bubble, the loop can be released from the bubble surface and move along its Burger's vector. This mechanism leads to enlargement of the bubble in the Burger's vector direction of the loop by extending the faces aligned with the slip planes, in which the loop sides glide. Thus, the helium bubble formation process has anisotropic nature due to existence of preferential directions associated with the crystal slip systems. The same bubble growth mechanism was observed in the simulations of the system at $3000 \mathrm{~K}$. Let us note that at sufficiently low temperatures tungsten exhibits brittle behavior leading potentially to nano-crystal cleavage by bubble induced stress. Although high concentrations of embedded helium can facilitate tungsten plasticity [13], the possibility of ductile to brittle transition in tungsten/helium nano-structures will be further investigated in our future work.

The bubble shape affects the elastic field created around it. In Fig.2 the tungsten atoms are shown on (111) plane, sliced through the sphere center, at the same bubble growth stage as in Fig.1. The color of the atoms is coding the local pressure, calculated as stress trace $-\operatorname{Tr}|\sigma| / 3$, with blue color corresponding to material compression (positive pressure) and red to tension (negative pressure). As we can see, the regions of strong material compression are adjacent to the bubble polyhedron faces, while the regions of tension are formed along the outer sides of dislocation curves and near the polyhedron vertexes. The maximum pressure in the compression regions is $p_{\max } \sim 60 \mathrm{GPa}$ at $1000 \mathrm{~K}$ and $p_{\max } \sim 30 \mathrm{GPa}$ at $3000 \mathrm{~K}$ that also 
corresponds to helium pressure in the bubble. The simulated maximum pressure values are directly related to the shear strength of tungsten at given temperatures for the used tungsten atomic potential [13], at which nucleation of the dislocations at the edges of the bubble polyhedron takes place and which can be estimated as $p_{\max } / 2$. Let us note, that the helium pressure in the bubble exceeds the pressure corresponding to the bubble surface tension, $p_{s}=2 \gamma_{s} / R$, even for the bubble radius $R$ of order of the lattice constant, where the tungsten surface tension coefficient is taken to be $\gamma_{s}=5.4 \mathrm{Jm}^{-2}$ [14]. The spatial extent of the highly stressed regions is of order of the bubble size in accord with the simple elasticity theory predictions for a spherical bubble. We also should note that the modelled strain rate during the bubble growth was very high due to high helium insertion rate limited by MD computational capabilities. However, additional simulations with interrupting the bubble growth and annealing the system for 10ns did not reveal any qualitative differences in the described above growth mechanism. Without sufficient helium pressure inside the bubble, the anchored dislocations could not be released and aside from slight elastic stress relaxation the bubble was almost static.

\section{Coalescence of helium bubbles in tungsten}

In order to study interaction of helium bubbles in tungsten, we simulated growth of two bubbles $1000 \mathrm{~K}$ from small nucleation volumes separated by distance $l=4 \mathrm{~nm}$ at $1000 \mathrm{~K}$. We used the same simulation technique, as described above, but instead of the sphere the bubbles were growing in a nano-cylinder of length $60 a \approx 18.99 \mathrm{~nm}$ and radius $25 a \approx$ 7.913nm. The nucleation points were placed on the cylinder axis, oriented along [111] direction, symmetrically relative to the middle point. In Fig. 3 the pressure profile around the two bubbles is shown on $(\overline{1} 10)$ plane, drawn through the cylinder axis, at the moment of the 
bubbles coalescence. As one can see, the both bubbles are non-spherical with their shapes approximating polyhedrons, as described in the previous section. The point of bubbles contact is not placed along the shortest connection length between their centers. At the moment of coalescence the sizes of the bubbles were $\sim 3 \mathrm{~nm}$ and the pressure in each of them was $\sim 60 \mathrm{GPa}$, the same as in the simulations of a single bubble growing at $1000 \mathrm{~K}$. During growth of the bubbles the pressure in them varied due to the dislocation loop punching process and increasing size of the bubbles. The loop punching lead to repeated peaking and quick relief of the pressure by several GPa, while the bubble growth caused gradual reduction of the pressure peaks due to decreasing of both the bubble surface tension and the strength of thinning tungsten shell. The bubbles form common stress field, which has a compression region in the space between the bubbles and in other aspects is qualitatively similar to the stress field around a single bubble (see Fig.2). The existence of the compression region between the bubbles indicates that the bubbles growth towards each other is energetically disadvantaged. It can also be understood from the point of view of interaction of dislocations produced by the bubbles. The growing towards each other bubbles would produce dislocation loops with Burger's vectors in same direction for same line sense that generally leads to repulsive interactions between the dislocations. Thus, coalescence of the bubbles largely happens by lateral growth, when the generated dislocations interact at larger angle between their Burger's vectors that is more energetically favorable according to Frank's rule.

In Fig.4, the tungsten atoms not in regular lattice positions are displayed for the same two bubble arrangement as in Fig.3, but at an earlier time moment. The complex stress field around the bubbles causes various reactions between the dislocations leading to formation of dislocations spanning from one bubble to another, even when they are not yet merged, see Fig.4. A very interesting feature observed in this figure is the formation of a vacancy tube by prismatic dislocation loop moving away from the bubbles. The tube is formed as not all 
dislocation segments start to move simultaneously in the complex stress field of the bubbles. If the resolved shear stress near one of the anchor points is not strong enough to release the loop, a long dislocation dipole of screw character is pinched from the point. Due to mutual attraction of the two dipole segments, having opposite line sense, the loop is eventually

released forming vacancy trail behind and possibly a jog on it. The trail can further break up to smaller segments by absorption/emission of point defects. The climb of the jog on the loop moving along its Burger's vector in the large stress field can also produce point defect debris, as seen in Fig.4. That leads to formation of vacancies aligned in the slip direction, which can serve as precipitation point for dissolved helium atoms and facilitate formation of chains of helium bubbles along $\langle 111\rangle$ crystallographic directions. Such mechanism may be responsible for helium bubble clusters observed in tungsten irradiated with D-He mixture plasma [15].

\section{Transport of helium atoms in stressed tungsten lattice}

The bubble growth in tungsten exposed to helium plasma should proceed by some transport mechanism of implanted helium atoms toward the bubble. To examine how helium interstitial atoms can diffuse in the stressed tungsten lattice surrounding bubbles, we performed simulations of helium atom dynamics in intentionally stressed tungsten. The monocrystal tungsten block of size $5 \times 5 \times 100$ lattice constants periodic in $\mathrm{X}$ [100] and Z [001] directions and having its $\mathrm{Y}[010]$ faces fixed at $\mathrm{Y}=-50 a$ and $50 a$ was simulated. The approximately uniform stress gradient of magnitude $\nabla \sigma \approx 10^{18} \mathrm{~Pa} / \mathrm{m}$ along the $\mathrm{Y}$ axis was created by applying force to each tungsten atom in -Y direction. Let us note, that pressure gradient in tungsten around a helium bubble may significantly exceed this value. For example, for the bubble in Fig. 2 with pressure $\sim 60 \mathrm{GPa}$ and size $\sim 2.5 \mathrm{~nm}$ it is $\sim 2.4 \times 10^{19} \mathrm{~Pa} / \mathrm{m}$. The diffusion of a single helium atom in this uniaxially strained system was simulated for different 
temperatures in the range $500-2500 \mathrm{~K}$. Total 30 different trajectories were simulated at each temperature for the duration of $100 \mathrm{~ns}$ or until the atom left the system. The helium diffusion coefficient, $D$, along $\mathrm{X}$ direction orthogonal to the applied force was obtained by linear fitting of time dependence of the helium atom square displacement in this direction for each trajectory and then calculating the mean value for a given temperature. The Arrhenius fit to the simulated diffusion coefficient dependence on temperature gives the helium diffusion activation energy $E_{d}=0.34 \mathrm{eV}$ and the pre-exponent factor $D_{0}=3.2 \times 10^{-8} \mathrm{~m}^{2} / \mathrm{s}$ in the whole temperature range. No significant drift of helium in the direction perpendicular to the applied stress was observed. However, the drift was apparent in $\mathrm{Y}$ direction of the stress gradient. The drift velocity, $V_{d}$, was calculated for each simulated temperature using method similar to the diffusion coefficient calculations. Assuming that the force acting on the helium atom is proportional to the stress gradient, we can write the drift velocity as $V_{d}=\gamma D \nabla \sigma / k_{B} T$, where $k_{B}$ is the Boltzmann constant and Einstein's relation for mobility, $\mu=D / k_{B} T$, is used. The proportionality coefficient $\gamma=3.3 \times 10^{-30} \mathrm{~m}^{3}$ is obtained from the simulations using the calculated values of $D$. For the bubble shown in Fig.2 with the estimated stress gradient $\nabla \sigma \sim 2.4 \times 10^{19} \mathrm{~Pa} / \mathrm{m}$ we obtain the drift velocity $V_{d} \sim 3.6 \mathrm{~m} / \mathrm{s}$ at $1000 \mathrm{~K}$. In this case, the drift would dominate helium transport on lengths larger than $D / V_{d} \sim 0.17 \mathrm{~nm}$, which is smaller than the lattice constant. Therefore, helium transport in the vicinity of the bubble is mainly governed by the formed stress field. As we saw in the previous sections, the stress field around the bubble has compression regions close to the bubble faces and tension regions covering the outer sides of dislocations and the bubble vertexes. As the helium atoms drift from the compression to the tension regions, we suggest that the dissolved helium can be transported along the dislocation lines towards the bubble vertexes, which serve as main gates of the helium penetration into the bubble. 


\section{Conclusions}

In the present work we investigated mechanisms of helium bubbles growth and coalescence in tungsten using molecular dynamics simulations. It was shown that the helium nano-bubbles in bcc tungsten have an irregular polyhedron shape with the faces generally parallel to the slip planes and the edges aligned with the $\langle 111\rangle$ slip directions. The mechanism of the bubble growth via formation, reaction and release of dislocations was described explaining the non-spherical shape of bubbles. It was shown that the helium pressure in the nano-bubble is determined largely by the shear strength of the crystal responsible for dislocation generation, while the surface tension plays lesser role even for the sub-nanometer bubbles. We demonstrated that the stress field formed around the bubble is anisotropic and has distinct compression and tension regions associated with the bubble faces and vertexes, respectively. The performed simulations of coalescence of two bubbles revealed that the merger proceeds by lateral growth of the bubbles, rather than along shortest connection length, even when the bubbles face each other along a slip direction. We also show that in the strong stress field around the bubbles incomplete release of a dislocation loop can lead to formation of long vacancy chains aligned in the slip direction. We suggest that helium precipitation on the vacancies can lead to formation of chains of helium bubbles along $\langle 111\rangle$ directions, which were observed experimentally. We also demonstrated that fast drift of helium atoms from compression to tension regions exists in the stressed lattice around the bubble. The helium transport coefficients in a stressed tungsten lattice were also determined. We argue that the drift dominates helium transport in the stress field around the bubbles causing helium to move along the dislocation lines towards the bubble vertexes, which play role of main locations for helium penetration into the bubble. The dislocations pinched by the bubbles also can trap and transport helium atoms as they slip and serve as precipitation locations for formation of new bubbles. We suggest that the described in this work dynamics 
of helium atoms and dislocations generated by the bubbles plays essential role in the fuzz formation. The further studies investigating microscopic fuzz growth mechanism will be presented in our future publications.

\section{Acknowledgements}

This work is performed under the auspices of the US DOE Grants Nos. DE-SC00-01999 and DE-SC00-08660 at UCSD. 


\section{References}

[1] S. Takamura, N. Ohno, D. Nishijima, and S. Kajita, Plasma Fusion Res. 1 (2006) 051.

[2] M.J. Baldwin and R.P. Doerner, Nucl. Fusion 48 (2008) 035001.

[3] S. Kajita, T. Saeki, N. Ohno, et al., J. Nucl. Mater. 417 (2011) 838.

[4] S.I. Krasheninnikov, Phys. Scr. T145 (2011) 014040.

[5] S.I. Krasheninnikov, T. Faney, and B.D. Wirth, Nucl. Fusion 54 (2014) 073019.

[6] S.J. Plimpton, J. Comput. Phys. 117 (1995) 1.

[7] http://www.ctcms.nist.gov/potentials/W.html

[8] N. Juslin and B.D. Wirth, J. Nucl. Mater. 432 (2013) 61.

[9] D.E. Beck, Mol. Phys. 14 (1968) 311.

[10] D.E. Beck, Mol. Phys. 15 (1968) 332.

[11] C.R. Krenn, D. Roundy, J.W. Morris Jr, and M.L. Cohen, Mater. Sci. Eng. A319-321 (2001) 111.

[12] A. Stukowski and K. Albe, Modelling Simul. Mater. Sci. Eng. 18 (2010) 085001.

[13] R.D. Smirnov and S.I. Krasheninnikov, Nucl. Fusion 53 (2013) 082002.

[14] E. Lassner and W.D. Schubert, “Tungsten: Properties, Chemistry, Technology of the Element, Alloys, and Chemical Compounds” (New York: Kluwer Academic, 1999) 15.

[15] M. Miyamoto, D. Nishijima, M.J. Baldwin, et al., J. Nucl. Mater. 415 (2011) S657. 


\section{Figure captions}

Fig.1 The simulated helium bubble containing 1000 atoms (red surface) and the displaced tungsten lattice atoms (gray circles) viewed along [111] crystallographic direction.

Fig.2 The simulated pressure profile on (111) plane in tungsten around the same helium bubble as in Fig.1.

Fig.3 The simulated pressure profile on (110) plane around the two helium bubbles (red surfaces) at the moment of coalescence.

Fig.4 The displaced tungsten lattice atoms (gray circles) around the two helium bubbles (red surfaces). 


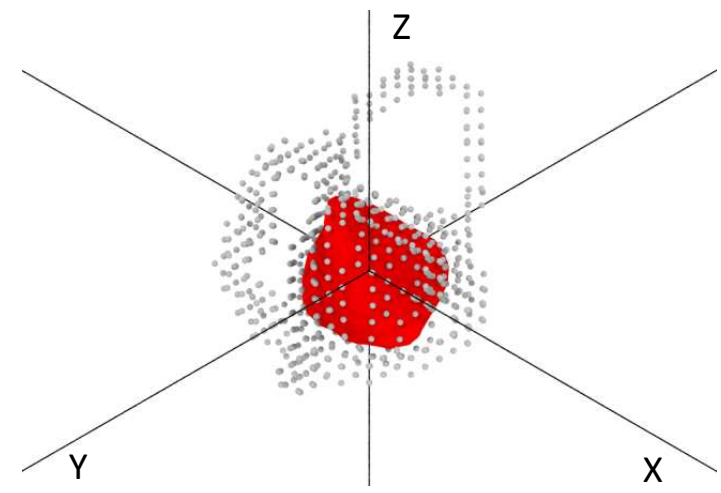

Fig.1 


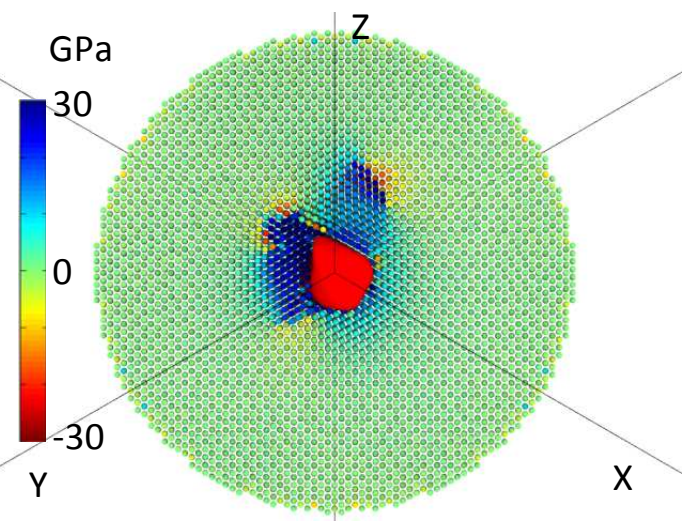

Fig. 2 


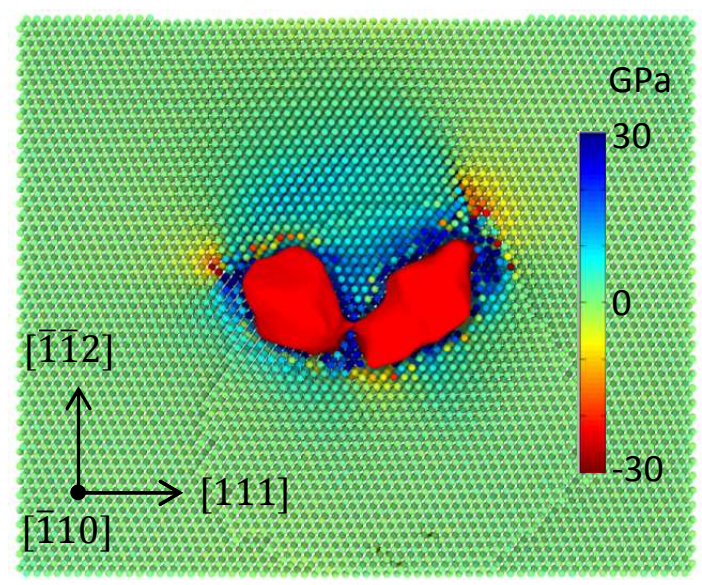

Fig.3 


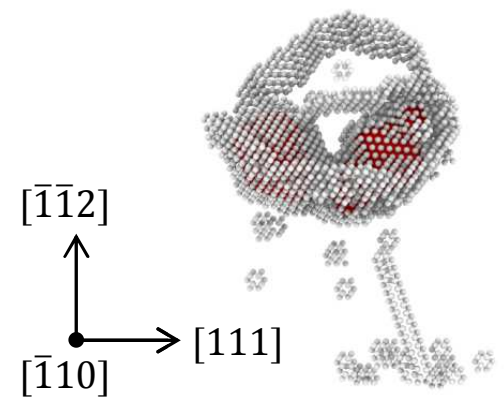

Fig.4 